\title{
ANÁLISIS ESTADÍSTICO SOBRE EL SISTEMA EDUCATIVO DEL ESTADO DE VERACRUZ
}

\author{
Teresa Zamora-Lobato \\ Instituto Tecnológico Superior de Misantla \\ maría.jz@misantla.tecnm.mx

\section{RESUMEN}

El documento ofrece un panorama general del sistema educativo veracruzano, con base en las estadísticas emitidas por la Secretaría de Educación de Veracruz. Se analizan documentos oficiales como el Plan Nacional de Desarrollo 2019-2024 y el Programa Sectorial de educación. Se utiliza una metodología analítica y descriptiva a través de recursos digitales e información documental. El análisis permite concluir la necesidad de realizar cambios estructurales para transformar a la educación con calidad a partir de rediseñar los planes y programas educativos, dotar de más y mejor infraestructura, capacitación a profesores y redefinir el perfil de egreso de estudiantes.

Palabras clave: alumnos; incremento; decremento; niveles

\section{ABSTRACT}

Statistical análisis of the education system in the state of Veracruz. The document provides an overview of the Veracruz education system, based on statistics issued by the Ministry of Education of Veracruz. Official documents such as the National Development Plan 2019-2024 and the Education Sector Program are analyzed. An analytical and descriptive methodology is used through digital resources and documentary information. The analysis allows concluding the need to make structural changes to transform education with quality by redesigning educational plans and programs, providing more and better infrastructure, training teachers and redefining the profile of students' graduation.

Keywords: students; increase; decrease; levels; increase; decrease. 


\section{ANÁLISIS ESTADÍSTICO SOBRE EL SISTEMA EDUCATIVO DEL ESTADO DE VERACRUZ}

\section{INTRODUCCIÓN}

En México la Ley General de Educación establece que existen tres tipos de educación: Educación Básica (preescolar, primaria y secundaria), Nivel Medio Superior y el Nivel Superior, mismo donde las escuelas y docentes también son un papel protagónico para el quehacer educativo. De igual forma, el servicio educativo que se brinda a la población se comprende los 212 municipios que integran el estado de Veracruz.

Para el presente trabajo de investigación la información recabada proviene de los planteles educativos públicos y privados del estado de Veracruz corresponde al periodo escolar 2018-2019 y está integrada en el Prontuario Estadísticos 2017-2018, en el cual está contenida la Información Estadística del Sistema Educativo Estatal donde también se consideran los niveles en apego al Esquema General del Sistema Educativo Nacional y desagregados por sus tipos y sostenimientos que se describen a continuación:

Dentro de la Educación Inicial comprenden las edades de niños que van desde los cuarenta y cinco días de nacidos hasta la edad de tres años, esta educación se brinda de manera escolarizada, no escolarizada e indígena.

La Educación Especial logra brindan sus servicios con recursos federales y estatales por medio de los Centros de Atención Múltiple y las Unidades de Servicio de Apoyo a la Educación Regular.

Posteriormente, se tiene la Educación Preescolar; la cual, abarca tres categorías: General, Indígena y CONAFE, con distinto sostenimientos como: estatal, federal, federalizado o particular.

Dentro de la Educación Primaria comprende las edades de entre seis y once años, cuenta con cuatro tipos de modalidades: General, Niños Migrantes, Indígenas y CONAFE, para este nivel se contempla la edad normativa de seis a once años y cuenta con cuatro tipos de sostenimiento, estatal, federal, federalizado y particular.

Además, dentro de los Institutos de Capacitación para el Trabajo del Estado de Veracruz (ICATVER) y Centros de Capacitación para el Trabajo Industrial (CECATI) que lo conforman 31 campos de formación profesional, 55 especialidades y 207 cursos, a través de estos, se da la Formación para el Trabajo, Educación Técnica y Desarrollo Productivo. ${ }^{1}$

Para la Educación Secundaria se cuentan con 138 de tipo urbano y 2,329 rurales, cuyas modalidades son: General, Para Trabajadores, Telesecundaria y Técnica. Además, cuenta con sus sostenimientos: federal, estatal, federalizado y particular.

Por su parte, para el componente Profesional Técnico, únicamente se contabilizan nueve escuelas en el estado de Veracruz con dos tipos de sostenimiento: estatal y particular.

En el Nivel Medio Superior o Bachillerato se cuentan con dos modalidades: General y Técnico. Las edades comprendidas en este nivel van entre los quince y diecisiete años de edad. Los sostenimientos se presentan de tres tipos: federal, estatal y particular.

Para el Técnico Superior Universitario, únicamente se contabilizan dos instituciones en el estado de Veracruz, las cuales, son de sostenimiento en sus dos modalidades; escolarizado y no escolarizado, existen dos Instituciones en el Estado y de sostenimiento particular.

Para la Educación Normal en el estado se cuenta con tres tipos de instituciones cuyos números son los siguientes: una escuela federal, cuatro estatales y once particulares en donde se imparten las licenciaturas de Preescolar, Primaria, Especial, Educación Física y Secundaria.

Asimismo, en la Licenciatura Universitaria y Tecnológica en el estado existe una Institución Autónoma, treinta estatales, dos federalizadas, dos federalizadas, nueve federales y ciento treinta particulares, en modalidades de tipo escolarizada y no escolarizada.

De igual modo, se cuenta con Posgrado Universitario y Tecnológico en modalidad tanto de tipo escolarizada como no escolarizada donde se imparte Especialidad, Maestría y Doctorado. Se contabilizan en el estado veinte instituciones y seiscientos doce programas con sostenimiento estatal, autónomo, federalizado y particular.

Además, en la Educación para Adultos el estado de Veracruz cuenta con sesenta instituciones donde se ofrece el servicio educativo a través de las escuelas de Educación Básica para Adultos (EBAS) de sostenimiento estatal y con sostenimiento federalizado las escuelas Misiones Culturales. 
Este documento de interpretación brinda una perspectiva y tiene el propósito de dar a conocer el comportamiento de los datos estadísticos del Periodo Escolar 2018-2019 en comparación con el periodo anterior respecto al aumento 0 disminución en la cantidad de escuelas, docentes, grupos, alumnos, así como localidades y municipios atendidos. Además, el porcentaje de distribución dentro del total del Sistema Educativo Estatal.

\section{METODOLOGíA}

Este trabajo se centra en el estudio de referencias para alcanzar resultados y conclusiones pertinentes relacionados con las cifras totales en los niveles educativos, comparando estadísticamente, entre ciclo 2017-2018 y 2018-2019. El estudio es etapas, la primera se basa en el análisis estadístico sobre los datos en los niveles educativos. La segunda etapa consiste en los análisis estadísticos del total por nivel educativo, enfocado al sostenimiento público.

\section{Análisis y discusión de datos}

Primeramente, en la Ley General de Educación se menciona que se establecen tres tipos de educación: Básica (se conforma de tres niveles: preescolar, primaria y secundaria), Media Superior y Nivel Superior, las cifras que el Sistema Educativo de Veracruz atiende incluyen 2, 235, 956 alumnos con 137,277 docentes en 102,325 grupos dentro de 24,036 escuelas. Aparte de ello, se brinda servicio a 6,828 localidades de los 212 municipios. En la tabla 1 se muestra el incremento 0 decremento de total de los datos estadísticos.

Tabla 1. Incremento o decremento del total de los datos estadísticos 911.

\begin{tabular}{|c|c|c|c|c|c|}
\hline Nivel/categoría & $\begin{array}{l}2017- \\
2018\end{array}$ & $\begin{array}{l}2018- \\
2019\end{array}$ & $\begin{array}{l}\text { Diferencia con } \\
\text { el periodo } \\
\text { anterior }\end{array}$ & $\begin{array}{l}\% \text { diferencia } \\
\text { con el } \\
\text { periodo } \\
\text { anterior }\end{array}$ & Variación \\
\hline Escuelas & 24119 & 24036 & -83 & -0.34 & Disminuye \\
\hline Docentes & 138880 & 137277 & -1603 & -1.15 & Disminuye \\
\hline Grupos & 103627 & 102325 & -1302 & -1.26 & Disminuye \\
\hline Alumnos & $\begin{array}{l}228575 \\
5\end{array}$ & $\begin{array}{l}223595 \\
6\end{array}$ & -49799 & -2.18 & Disminuye \\
\hline Municipios & 212 & 212 & 0 & 0.00 & Igual \\
\hline Localidades & 6875 & 6828 & -47 & -0.68 & Disminuye \\
\hline
\end{tabular}

Para su cálculo se tomaron los datos de los Prontuarios Estadísticos (Inicio Ciclo Escolar 2017-2018 y 2018-2019)

\section{Fuente: SEV/UPECE; Estadística Educativa}

Al comparar los datos estadísticos, en la figura 1, se observa que durante el Periodo 2018-2019 hubo un decremento en todos los aspectos al comparar con el periodo anterior inmediato 2017-2018, observando una diferencia de 49,799 Alumnos, menos, simbolizando el -2.18\%, cabe destacar que dicho decremento se viene arrastrando en periodos anteriores.

Algunos de los factores que contribuyen al abandono en cualquier nivel educativo, no es por cuestiones económicas, ni en el modelo educativo, es más hacia factores como la depresión, el déficit de atención, la hiperactividad, la migración, la planificación familiar, entre otros (Fortin, Marcotte, Potvin, Poyer y Joly, 2006; Fortin, Royer, Potvin, Marcotte y Yergeau, 2004; Janosz, Archambault, Morizot y Pagani, 2008; Lessard, Buttler-Kisber, Fortin, Marcotte, Potvin y Royer, 2008; González, 2006). 


\section{ANÁLISIS ESTADÍSTICO SOBRE EL SISTEMA EDUCATIVO DEL ESTADO DE VERACRUZ}

Asimismo, se contempla una disminución de 1302 referente, a los Grupos atendidos constituyendo el $1.26 \%$ sin embargo, se observa igual un descenso de 1,603 docentes expresando el -1.15\%, y esto es lógico porque se observa la baja de 83 escuelas aludiendo el $-0.34 \%$.

De acuerdo con Botello-Peñaloza muestra relación negativa entre el tamaño de la clase y el desempeño académico en América Latina con base en la prueba internacional PISA de 2012 (2016, p. 106), significando que, los grupos reducidos poseen ventaja, entre ellas: existe una mayor participación de los educandos. Además, se genera una relación personalizada docente-alumno y la focalización educativa con base al entorno social de los estudiantes.

En contraste, la Organización para la Cooperación y el Desarrollo Económicos (OCDE) señala que "en conjunto no hay pruebas determinantes acerca de la repercusión del tamaño de las clases en el rendimiento de los estudiantes" (p. 418).

Además, referente a las Localidades atendidas, se observa una disminución de 47 unidades, cuyo porcentaje representa el -0.68\% al verificarlo con el periodo preliminar. No obstante, se cubrió la atención en los 212 municipios, los mismos del ciclo anterior inmediato.

Figura 1. Incremento o decremento del total de los datos estadísticos 911.

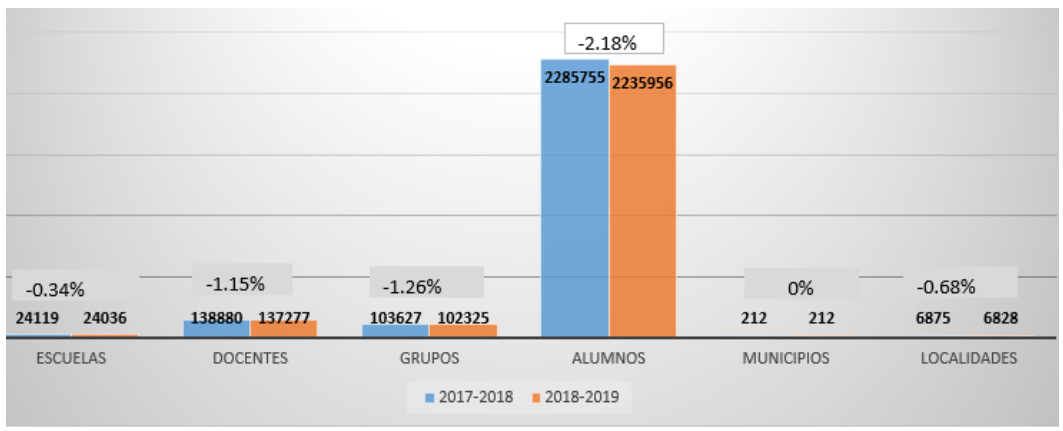

Para su cálculo se tomaron los datos de los Prontuarios Estadísticos (Inicio Ciclo Escolar 2017-2018 y 2018-2019)

Fuente: SEV/UPECE; Estadística Educativa.

\section{Incremento o decremento de escuelas por Nivel Educativo}

A través del análisis sobre la comparación e interpretación de los datos estadísticos que se presentan en la figura 2, se observa que la cantidad de escuelas en el Periodo Escolar 2018-2019 disminuyó en seis niveles educativos; tales como: Educación Inicial donde, se muestra una diferencia de $-38.24 \%$ en cuanto, a Educación Primaria, se observa un contraste de $-0.55 \%$.

Sin embargo, con referencia a Formación para el Trabajo se visualiza $-1.51 \%$. De igual modo, en las Normales muestra el -6.25\%, Licenciaturas (Universidad y técnico superior) se observa -0.58\%, y, por último, se visualiza Educación para Adultos, con una disminución del -1.53\%.

Por lo anterior, cabe destacar que es evidente por la tendencia que se debe entre otros factores a la disminución en la población en ese rango de edad. A manera de ejemplo, de acuerdo con el análisis de la estadística reportada en los ciclos escolares anteriores, 2017-2018 y 2018-2019, con base a los cuestionarios de la 911 , señala que el municipio de Álamo, presenta una disminución en la matrícula de un 2.8\% para primaria y un 3.4\% para preescolar, siendo una matrícula menor a 30 alumnos.

Por el contrario, los cuatro niveles que presentaron un aumento se señalan a continuación: Educación Especial con $0.90 \%$ escuelas más que el año anterior. Lo anterior, es evidente debido a que el comportamiento de dicho nivel en los rubros que se trabajan en este documento, se percibe un crecimiento, siguiéndole, 
Educación Preescolar con una participación de $0.74 \%$ planteles más, con una intervención minúscula. A continuación, se muestran, Secundarias con $0.84 \%$, en cuanto a Bachillerato se percibe un crecimiento de $0.55 \%$ planteles, no obstante, Profesional técnico, Técnico superior Universitario y Posgrados; se mantuvo la misma cantidad de escuelas.

Figura 2. Incremento/ decremento de escuelas por nivel educativo periodo 2017-2018 y 2018-2019.

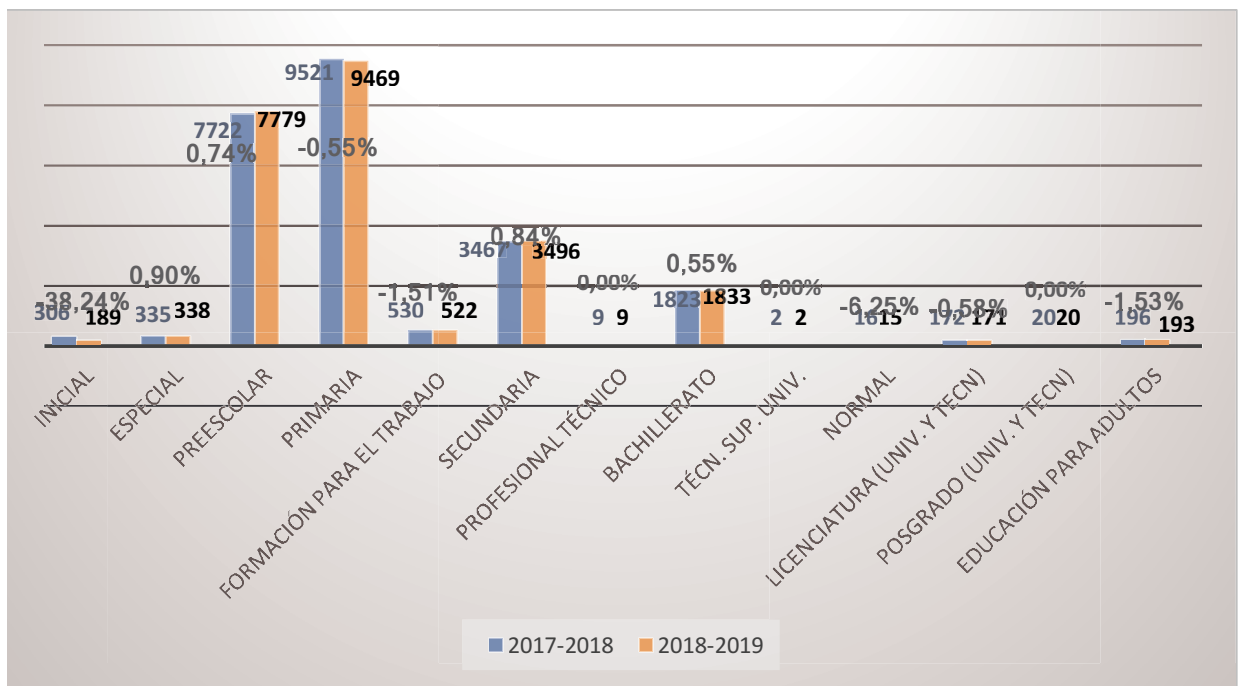

Fuente: SEV/UPECE; Estadística Educativa.

Cabe mencionar que la cantidad de escuelas se contabiliza en función al turno y no al inmueble escolar. Asimismo, en Educación Normal y Superior se contabiliza por institución. En las instituciones de Técnico Superior Universitario están contabilizadas dentro de la Educación Superior.

\section{Incremento o Decremento de Docentes por Niveles Educativos}

En la figura 3 se comprende que el número de docentes que incrementaron en dicho periodo, corresponden a 6 niveles Educativos: en primer lugar, se observa Educación Especial reflejando el 3.35\%, al igual se observa la Educación Preescolar con una variación a favor de $0.10 \%$.

En nivel Primaria se visualiza el $0.18 \%$, por otro lado, nos encontramos con el rubro de Profesional Técnico y Posgrado Universitario y Tecnológico muestra a favor el 3.70\%, sin embargo, el más notorio es el aumento de $363 \%$ en Técnico Superior Universitario, y esto parece evidente porque más adelante se observa que se incrementó la cantidad de municipios atendidos igualmente, para la Educación Normal señala el 12.21\% más que el año pasado.

Caso contrario, con los siguientes niveles, los cuales enunciaremos en los que hubo Decremento, comparando 2018-2019 con el periodo anterior inmediato, comenzamos con Educación Inicial, el cual, nos muestra, el -72.49\%, cabe señalar, mencionar que la justificación para asignar los recursos obedece a un análisis basado en los criterios técnico-normativos, plantilla de personal y la estructura ocupacional vigente; en este sentido, puede darse el caso que en muchos de los centros de trabajo que se exige personal, éste no se justifique, toda vez que presenta superávit u óptimo docente. De otra manera, se atiende la necesidad con la optimización de recursos o el incremento, siempre y cuando exista suficiencia presupuestal. 


\section{ANÁLISIS ESTADÍSTICO SOBRE EL SISTEMA EDUCATIVO DEL ESTADO DE VERACRUZ}

Al mismo tiempo, en Formación para el Trabajo, muestra el mismo efecto con el -6.96\%, por su parte, en Secundaria fue pequeño con el $-0.80 \%$, siguiéndole, Bachillerato con el $-1.75 \%$, de igual modo, se muestra la Licenciatura Universitaria y Tecnológica, con una mínima variación del -0.07\%, y en último lugar nos encontramos con Educación para Adultos donde su disminución es del -5.55\%. Ver figura 3.

Figura 3. Total de docentes por nivel educativo

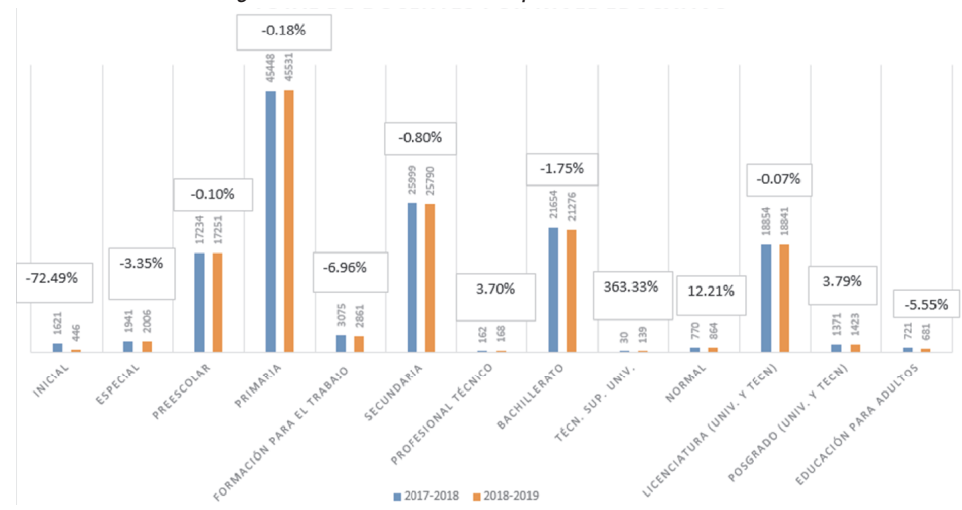

Fuente: SEV/UPECE; Estadística Educativa.

\section{Incremento o Decremento de Grupos por Nivel Educativo}

Los Grupos se entiende por el conjunto de alumnos que cursan, en un mismo espacio educativo y con igual horario, las materias o cursos establecidos en un plan o programa de estudios correspondiente a un grado escolar. En el caso de preescolar, el programa de estudios no contempla una estructura por asignaturas sino por campos formativos y competencias relacionadas con los mismos (SEP, 2018).

Sin embargo, en la figura 4 se observan, Ios niveles que tienen un Incremento tales como Educación Especial con un $6.69 \%$, y Profesional Técnico con el $10.26 \%$, sin duda son los niveles que más acrecentamiento tuvo y es obvio porque el comportamiento que tuvieron en este periodo, en comparación con el ciclo anterior inmediato, crecieron en varios aspectos, que conciernen a los rubros trabajados en este documento, por su parte, Preescolar refleja el $0.29 \%$, siguiéndole, Primarias figurando con el $0.20 \%$, todo esto, concerniente al tema de aumento de grupos durante el Periodo Escolar 2018-2019.

Figura 4. Total, de grupos por nivel educativo

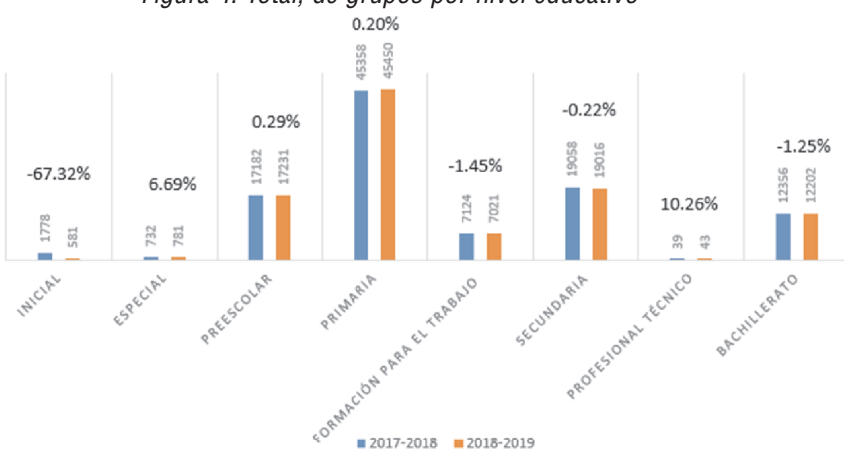

Fuente: SEV/UPECE; Estadística Educativa. 
Por el contrario, 4 niveles tuvieron un Decremento, tal como Educación Inicial con el -67.32\%, asimismo, en Formación para el Trabajo señala el $-1.45 \%$, continuando con, Secundarias muestran el $-0.22 \%$, y por último se visualiza bachillerato con el $-1.25 \%$.

Sin embargo, de acuerdo a los cuestionarios de la 911, en diversas escuelas, se redujo los grupos, debido a que administrativamente se realizó una compactación, es decir, los grupos del turno vespertino se trasladó al matutino, con el fin de mejoras en el comportamiento y el aprendizaje, esto con la finalidad de desocupar aulas que son las que representan el mayor riesgo, para los alumnos, debido a las contingencias naturales que se han presentado actualmente (Illuvias, temblores, deslaves, etc.).

Cabe destacar que Técnico Superior Universitario, Normales, Licenciatura (Universidades y Tecnológicos), Posgrados (Universidades y Tecnológicos) y Educación para adultos no reportan Información sobre grupos, debido a que tienen un programa deferente, ellos manejan por semestres o cuatrimestres y los otros niveles, son por año completo.

\section{Incremento o decremento de Alumnos por Nivel Educativo}

El alumno es la persona matriculada en cualquier grado de los diversos tipos, modalidades y servicios educativos del Sistema Educativo Nacional (SEP 2018).

Primeramente, el que tuvo una Disminución con gran significancia es Educación Inicial del -67.87\%, a pesar de estas bajas tasas de matrícula, en la educación inicial, en Veracruz existen grupos numerosos de niños por personal educativo.

Por su parte en Educación Preescolar se observa el -2.56\%, siguiéndole, Primarias $-1.30 \%$, igualmente, se muestra, Formación para el Trabajo con el -2.82\%, además, Educación Secundaria señala -2.80\%, asimismo, Bachillerato, simboliza el $-1.19 \%$, por su parte, Técnico Superior Universitario es del $-5.03 \%$, para finalizar, se muestra Educación Normal con el -7.35\% (ver figura 5).

No obstante, en cinco niveles, presentaron un Crecimiento; en particular, se visualiza, Educación Especial con el $\mathbf{0 . 9 9 \%}$, en cuanto, a Profesional técnico, señala el 5.75\%, con respecto, a Licenciatura Universitaria y Tecnológica figura el $0.53 \%$, sin embargo, Posgrados Universitaria y Tecnológica, reflejan el 0.10\%, con relación a Educación para Adultos, simbolizan el $20.06 \%$, sin duda éste es el más alto debido a que actualmente existen varios programas para que puedan acceder a la educación, tal como Instituto Nacional para la Educación de los Adultos (INEA), a través de los Institutos Estatales de Educación para los Adultos (IEEA), y, Modelos de Transición Escolarizados (Modelo Pedagógico de Educación Primaria para Adultos -MPEPA- y Secundaria Abierta -SECAB), al igual, diseñó y administró el Modelo de Educación para la Vida y el Trabajo (MEVyT), Consejos Técnicos de Educación para Jóvenes y Adultos (CTEJA's), entre otros.

Figura 5. Total, de alumnos por nivel educativo

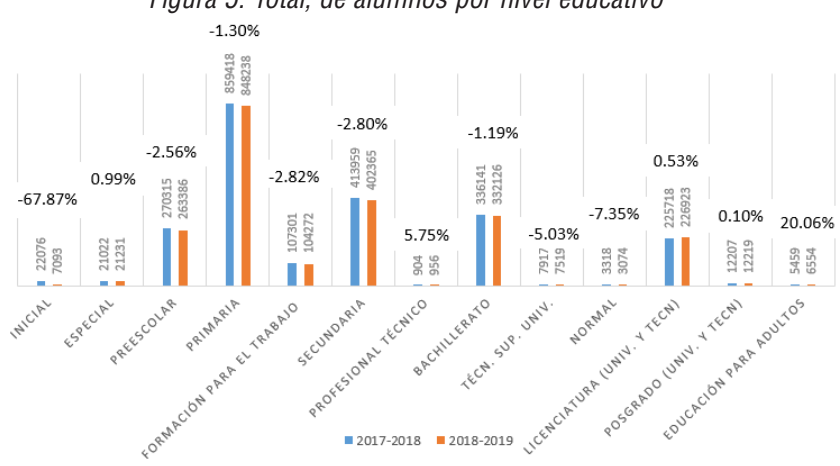

Fuente: SEV/UPECE; Estadística Educativa. 


\section{ANÁLISIS ESTADÍSTICO SOBRE EL SISTEMA EDUCATIVO DEL ESTADO DE VERACRUZ}

\section{Incremento o decremento de Municipios Atendidos por Nivel Educativo}

Los niveles en Educación Especial, Preescolar, Primaria, Formación para el Trabajo, Profesional Técnico, Secundaria; Bachillerato. y Normal, cubrieron el 99\% de los municipios, lo contrario a Educación Inicial disminuyó, el -55\%, es decir, no atendieron a toda la población, esto es lógico debido al cierre de guarderías que se registraron en los cuestionarios de la 911, cabe destacar, que a nivel nacional se han registrado diversas eventualidades ocurridos en educación inicial, pero, gracias a la preocupación de las autoridades, revisando la mala infraestructura evitando y previniendo mediante leyes que obliguen el establecimiento de un mínimo de condiciones de funcionalidad (Mateo-Díaz, Rodríguez-Chamussy \& Grafe, 2014). Por su parte, Educación para Adultos con el $-4 \%$.

Sin embargo, Técnico Superior. Universitario, incrementó el 0.9\%, a la par, Licenciatura (Universidades y Tecnológicos) con el 2\%, siguiéndole, Posgrados (Universidades y Tecnológicos) figurando el 3.45\%, sin duda es un gran avance, ya que se reactivaron las becas nacionales para la educación superior (Veracruz, 2019). Ver figura 7.

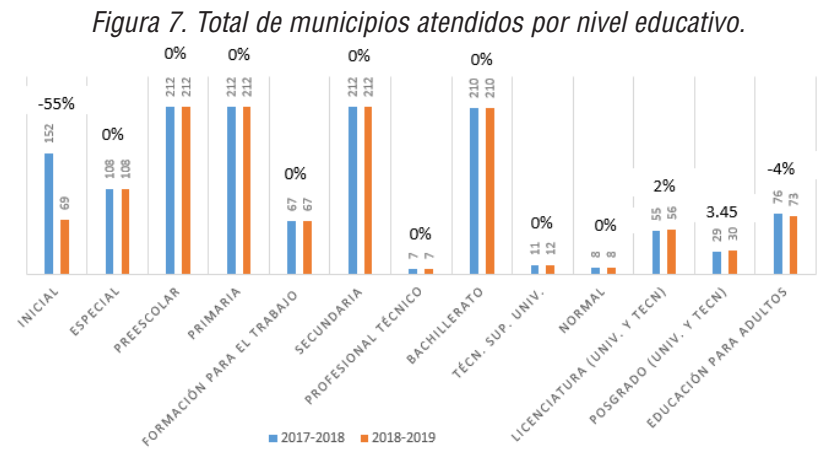

Fuente: SEV/UPECE; Estadística Educativa.

\section{Incremento o decremento del número de Localidades atendidas por Nivel Educativo}

De acuerdo, a la encuesta Intercensal realizada por el Instituto Nacional de Estadística y Geografía (INEGI, 2015), dentro del Estado de Veracruz existen 27,545 localidades donde el 25\% son atendidas. Asimismo, es necesario que se analicen las características de cada una de ellas para identificar las que ameritan o no la creación de planteles escolares.

Como se observa en la figura 8, el $46.15 \%$ de los niveles disminuyeron en la prestación de servicios en la cantidad de localidades; $23.08 \%$ se mantuvieron igual y el $30.77 \%$ aumentaron su cobertura.

Por otro lado, pasando al tema sobre el Sostenimiento, antes que nada, este se define como la fuente que proporciona los recursos financieros para el funcionamiento del centro de trabajo.

Por consiguiente, dentro del sistema de centros de trabajo se utilizan diferentes tipos de sostenimientos tales como: federal, estatal, municipal, autónomo y particular y subsidiado. A continuación, se muestra la interpretación de los niveles en apego al Esquema General del Sistema Educativo Nacional de acuerdo a su tipo y sostenimiento, mediante, su financiamiento, es decir, los recursos económicos, producto de los esfuerzos presupuestarios del sector público, del particular y de los fondos provenientes de fuentes externas, destinadas a financiar las actividades del Sistema Educativo Nacional. 
Figura 8. Total de localidades atendidas por nivel educativo

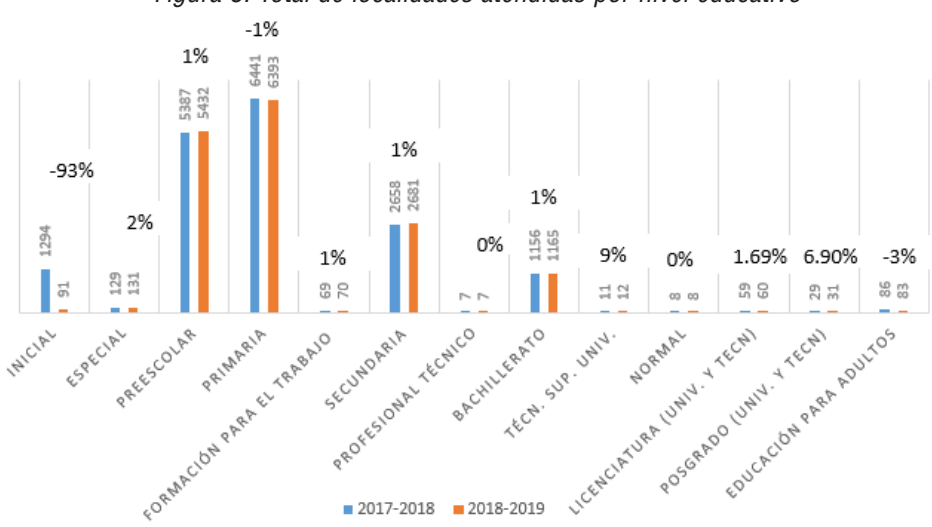

Fuente: SEV/UPECE; Estadística Educativa.

Cabe resaltar que la información que se presenta en este trabajo, la proporcionan directamente de las escuelas públicas y privadas del Estado de Veracruz al inicio del ciclo escolar 2018-2019, mediante los cuestionarios de la 911, a partir de un sistema de información coordinado por la SEV. Los datos generados brindan la información estadística oficial del gobierno federal y estatal.

Además, los indicadores que aparecen en el documento, se formulan a partir de la información estadística educativa y de las proyecciones de población que elaboró el Consejo Nacional de Población (CONAPO) proyecciones (2018).

Por lo que se refiere, al Sistema Educativo del Estado de Veracruz cuenta con 21467 escuelas por sostenimiento público, en comparación con Periodo Escolar anterior inmediato 2017-2018 señala 21569 presentando un decremento general del $-0.47 \%$.

\section{Incremento o decremento de escuelas por sostenimiento público y Nivel Educativo}

En la figura 9, se muestra que el periodo, se distinguió por la disminución en colegios, principalmente en Educación Inicial la cual decreció -54\%, siguiéndole, licenciatura (Univ. y TECN.) con -5\%, de modo semejante, formación para el trabajo simbolizando, con el -3\%, igualmente, Educación para adultos figurando con el -2\%, siguiéndole Educación primaria con el -1\%.

Por el contrario, Posgrado (Universidades y tecnológicos), despuntó con incremento del $50 \%$ en escuelas, sin embargo, Educación especial, Preescolar y Secundaria, se observa una progresión del 1\%, finalmente Bachillerato, tuvo una significancia del $0.21 \%$.

Los niveles de Profesional Técnico, Técnico Superior Universitario, Educación Normal, se mantuvieron sin ninguna variación. 


\section{ANÁLISIS ESTADÍSTICO SOBRE EL SISTEMA EDUCATIVO DEL ESTADO DE VERACRUZ}

Figura 9. Incremento o decremento de docentes por sostenimiento público y Nivel Educativo

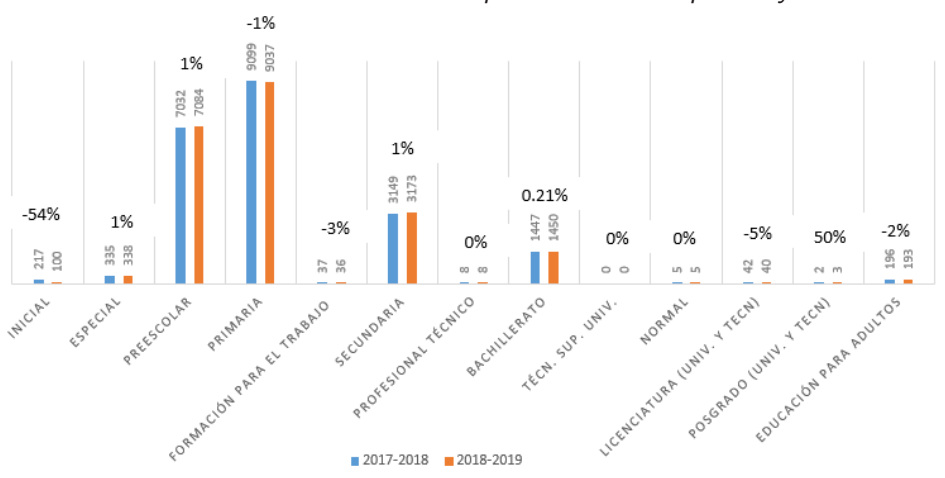

Fuente: SEV/UPECE; Estadística Educativa.

\section{Incremento o decremento de docentes por sostenimiento público y Nivel Educativo}

Los docentes son parte fundamental dentro del Sistema Educativo en Veracruz, debido a que son un factor fundamental en el aprendizaje de los estudiantes.

A continuación, en la gráfica 10, se observa una Disminución, en 6 niveles tales como Inicial, mostrando en el ciclo 2018-2019, con 192 docentes, siendo que, en el periodo anterior, estaba conformado por 1,376 maestros arrojando una diferencia de -1184 simbolizando el -86\% y esto es lógico porque a lo largo del trabajo se ha mostrado múltiples factores de dicha baja.

Por su parte en formación para el trabajo, expone 770 profesores en el periodo 2018-2019, siendo que, en el ciclo anterior, estaba formado por 844 docentes, generando un resultado de -74, el cual, representa el $-0.9 \%$.

En cuanto, a bachillerato, presenta 16,069 catedráticos en el periodo 2018-2019, presenciando que, en el ciclo anterior, contaba con 16,393 docentes, concibiendo un resultado de -324, representando el $-1.98 \%$.

Por su parte, licenciaturas (Universidades y tecnológicos), ostentando 10,546 pedagogos en el periodo 2018-2019, reflejando que, en el ciclo anterior, detallaba 11049 docentes, reconociendo una diferencia de -503, simbolizando el -5\%, siguiéndole, educación para adultos, el cual, manifiesta 681 educadores en el periodo 2018-2019, expresando que, en el ciclo anterior, enumeraba 721 docentes, registrando una diferencia de -40, simbolizando el $-6 \%$.

Por último, se contabiliza en secundarias a 21,998 pedagogos en el Periodo 2018-2019. En contraste, con el ciclo anterior, detallaba 2,293 docentes, registrando una diferencia de -295, simbolizando el -1\%.

Por otro lado, también se observa un Aumento, en 7 niveles tales como especia/ revelando en el ciclo 20182019, con 2,006 maestros, sin embargo, en el periodo anterior, estaba atendido por 1941 docentes, proyectando un beneficio de 65 pedagogos, simbolizando el 3\%, por su parte preescolar, presenta 15,292 profesores en el periodo 2018-2019, al cotejar, con el ciclo anterior, se visualiza 15,289 docentes, dando un resultado de 3 a docentes a favor, significando el $0.02 \%$, en cuanto, a primarias, se presenta 42,918 catedráticos en el periodo 2018-2019, sin embargo, en el ciclo anterior, contaba con 42,859 docentes, obteniendo un resultado de 59, éste mismo representando el $0.14 \%$.

Siguiéndole, profesional técnico, el cual, manifiesta 163 educadores en el periodo 2018-2019, pronunciando que, en el ciclo anterior, puntualizaba 159 docentes, al comparar ambos se visualiza 4 docentes más, simbolizando el 3\%, en cuanto a Técnico Superior Universitario, tuvo un gran aumento del 718\%, debido a que en este ciclo, obtuvo 90 pedagogos más que en el periodo pasado, el cual se visualiza 11 dando una diferencia a favor de 79 profesores, siguiéndole Educación Normal, generando 114 pedagogos a favor, puesto que, en el ciclo pasado se obtuvo un conteo de 565 maestros, siendo que en este ciclo se visualiza 679 docentes, simbolizando el 
$20 \%$ de la diferencia generada, para finalizar, se presenta Posgrado (Universidades y tecnológicos), expresando el $6 \%$ de aumento en docentes, debido a que la diferencia a favor fue de 49 pedagogos, ya que, en el ciclo 20182019 refleja 818 maestros y en el periodo anterior se contabilizo 769 educadores.

Figura 10. Incremento o decremento de docentes por sostenimiento público y Nivel Educativo.

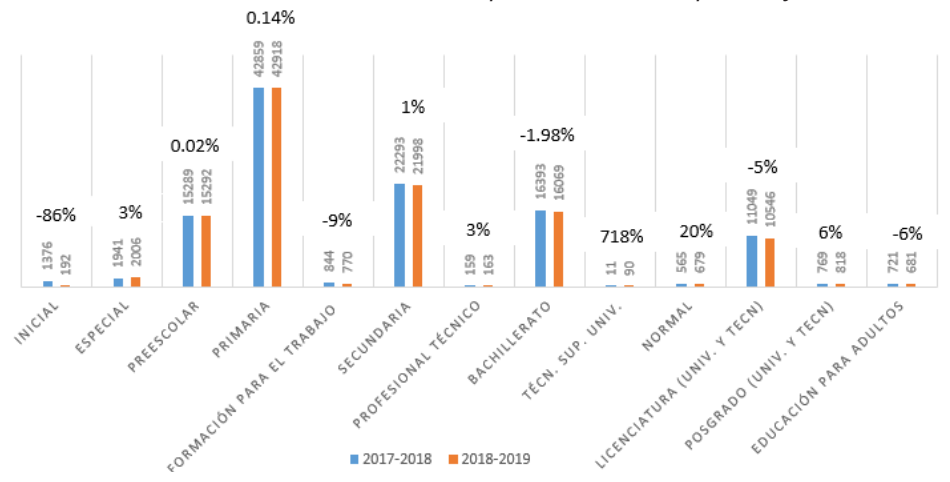

Fuente: SEV/UPECE; Estadística Educativa.

\section{CONCLUSIONES}

Como se pudo observar a lo largo de este trabajo, existe una tendencia de decrecimiento del $0.034 \%$ en las escuelas en diferentes niveles educativos como Educación Inicial, Educación Primaria, Formación para el trabajo, Educación Normal, Licenciatura (Universidades y Tecnológicos) y Educación para Adultos del Sistema Educativo del Estado de Veracruz correspondiente al Periodo Escolar 2018-2019. Por otro lado, Ios niveles que se resultado beneficiados se encuentran: la Educación especial, Preescolar, Secundaria y Bachillerato.

Con respecto al número de docentes se ha observado un decremento global del 1.15\%, resultado del comportamiento estadístico de los niveles de Educación Inicial, Formación para el trabajo, Secundaria, Bachillerato, Licenciatura Universitaria y Tecnológica, y Educación para Adultos, siendo beneficiados Educación especial, preescolar, primarias, profesional técnico, Técnico Superior Universitario, Normales, Posgrado (Universidades y tecnológicos).

Asimismo, en el comparativo realizado del Periodo Escolar 2018-2019, con respecto al anterior, se observó un decremento general del 1.26\% en la cantidad de grupos. No obstante, en los Niveles de Educación Especial, Preescolar, Primarias, Profesional Técnico se presentaron incrementos en la cantidad de grupos.

Además, la población estudiantil total de este Periodo Escolar disminuyó en 2.18\%; Ios Niveles Educativos afectados son: Educación Inicial, Especial, Preescolar, Primaria, Formación para el trabajo, Secundaria, Bachillerato, Técnico Superior Universitario y Normal.

\section{Contribuciones a futuraslíneas de investigación}

Como futuras líneas de investigación se propone que, los datos contenidos, permitirán profundizar en las diferencias existentes entre los trabajos realizados en el nivel educativo, ya sea: inicial, primaria, secundaria, bachillerato, licenciatura y el posgrado en aspectos como el enfoque metodológico, las temáticas, investigación cualitativa sobre los incrementos y decrementos en cada nivel, se puede visauliazar la demanda que obtendrá el nivel educativo, en el ciclo escolar inmediato. 


\section{ANÁLISIS ESTADÍSTICO SOBRE EL SISTEMA EDUCATIVO DEL ESTADO DE VERACRUZ}

\section{REFERENCIAS BIBLIOGRÁFICAS}

Botello-Peñaloza, H. A. (2016). Desempeño académico y tamaño del salón de clase: evidencia de la prueba PISA 2012. Actualidades Pedagógicas, 1(67), 97-112.

Fortin, L., Marcotte, D., Potvin, P., Royer, É., \& Joly, J. (2006). Typology of students at risk of dropping out of school: Description by personal, family and school factors. European Journal of Psychology of education, 21(4), 363-383.

Fortin, L., Royer, É., Potvin, P., Marcotte, D., \& Yergeau, É. (2004). La prediction du risque de decrochage scolaire au secondaire: facteurs personnels, familiaux et scolaires. Canadian Journal of Behavioural Science/Revue canadienne des sciences du comportement, 36(3), 219.

Gázquez, J. J., del Carmen Pérez, M., Ruiz, M. I., Miras, F., \& Vicente, F. (2006). Estrategias de aprendizaje en estudiantes de enseñanza secundaria obligatoria y su relación con la autoestima. International Journal of Psychology and Psychological Therapy, 6(1), 51-62.

México, DF: SEP. (2008). Glosario. Términos utilizados en la Dirección General de Planeación y Programación. Recuperado de http://cumplimientopef.sep.gob.mx/2010/Glosario\%202008\%2024-jun-08.pdf

Gázquez, J. J., del Carmen Pérez, M., Ruiz, M. I., Miras, F., \& Vicente, F. (2006). Estrategias de aprendizaje en estudiantes de enseñanza secundaria obligatoria y su relación con la autoestima. International Journal of Psychology and Psychological Therapy, 6(1), 51-62.

Juárez, D. B. (2018). Estudio Diagnóstico del Derecho a la Educación 2018. Primera edición: noviembre de 2018 Consejo Nacional de Evaluación de la Política de Desarrollo Social Av. Insurgentes Sur 810.

Lessard, A., Butler-Kisber, L., Fortin, L., Marcotte, D., Potvin, P., \& Royer, É. (2008). Shades of disengagement: High school dropouts speak out. Social psychology of education, 11(1), 25-42.

Lessard, A., Poirier, M., \& Fortin, L. (2010). Student-teacher relationship: A protective factor against school dropout?. Procedia-Social and Behavioral Sciences, 2(2), 1636-1643.

Mateo Díaz, M., Rodriguez Chamussy, L., \& Grafe, F. (2014). Ley de Guarderías en México y los desafíos institucionales de conectar familia y trabajo.

OCDE. (2016). Panorama de la Educación 2016. Indicadores de la OCDE. Madrid: Santillana.

Xalapa: SEV. (2018). Prontuario Estadístico Inicio de Cursos 2017-2018. Recuperado de https://www.sev.gob.mx/upece/blog/estadistica-educativa/

Xalapa: SEV. (2019). Prontuario Estadístico Inicio de Cursos 2018-2019. Recuperado de https://www.sev.gob.mx/upece/blog/estadistica-educativa/ 